\title{
Qualitative Analysis of Nonconvolution Volterra Summation Equations
}

\author{
Youssef N. Raffoul \\ University of Dayton \\ Department of Mathematics \\ Dayton, Ohio, USA
}

\begin{abstract}
This paper is first in a series of papers in which we consider the vector nonconvolution Volterra summation equation

$$
x(t)=a(t)-\sum_{s=0}^{t-1} C(t, s) x(s), t \in \mathbb{Z}^{+}
$$

where $x$ and $a$ are $k$-vectors, $k \geq 1$, while $C$ is an $k \times k$ matrix. Fixed point theorem, combined with resolvent and Lyapunov functionals are utilized to obtain conditions for boundedness of solutions, the existence of asymptotically periodic solution and the decay of solutions to zero.
\end{abstract}

AMS Subject Classifications: 34D20, 34D40, 34K20.

Keywords: Volterra summation equation, Boundedness, Lyapunov functional, Resolvent, Asymptotically periodic.

\section{Introduction}

We begin by stating some notations that will be used throughout this paper. For integers $a$ and $b$, we denote $[a, b]=\{a, a+1, \cdots, b\}$ and $\sum_{s=a}^{b} x(s)=0$ for $a>b$. Volterra summation equations play a major role in the qualitative analysis of neutral difference equation. To see this, we let $C$ to be the set of sequences $\phi:[-\alpha, 0] \rightarrow \mathbb{R}, \alpha>0$. Let

Received November 19, 2018; Accepted February 12, 2019

Communicated by Mehmet Ünal 
$\triangle$ be the forward difference operator and let $D$ be continuous in the second argument such that $D: \mathbb{Z} \times C \rightarrow \mathbb{R}$ and consider the neutral difference equation

$$
\triangle\left(D\left(n, x_{n}\right)\right)=f\left(n, x_{n}\right), \quad n \in \mathbb{Z}^{+}
$$

where, $x_{n}(s)=x(n+s), s \in C$. If

$$
D(n, 0)=f(n, 0)=0,
$$

then one would have to ask that stability of the zero solution of $D(n, 0)=0$ in order for the stability of the zero solution of (1.1). On the other had, if $h$ is a given sequence and we are interested in studying boundedness of solutions of (1.1), one would have to require that solutions of

$$
D\left(n, x_{n}\right)=z(n),
$$

be bounded for a suitable function $z(n)$ with suitable conditions. Equation (1.1) is typified by neutral equations of the form

$$
\triangle\left(x(n)+\sum_{s=n-h}^{n-1} b(s+h) x(s)\right)=f\left(n, x_{n}\right) .
$$

For more on neutral difference equations, we refer to [2] and [13]. We consider the vector Volterra summation equation

$$
x(t)=a(t)-\sum_{s=0}^{t-1} C(t, s) x(s), t \in \mathbb{Z}^{+}
$$

where $x$ and $a$ are $k$-vectors, $k \geq 1$, while $C$ is an $k \times k$ matrix. To clear any confusion, we note that the summation term in (1.3) could have been started at any initial time $t_{0} \geq 0$. The contents of this paper is considered to be of introductory level. However, the methods that we use in here are totally different than those of any published results. As we have mentioned in the abstract, this paper will be the first in a series that this author is undertaking to fully study Volterra summation equations. In our analysis will use the resolvent equation that was established on time scales in [1], combined with Lyapunov functionals and fixed point theory to obtain boundedness of solutions and their asymptotic behaviors. One of the major difficulties when using a suitable Lyapunov functional on Volterra summation equation is relating the solution back to the Lyapunov functional. For $x \in \mathbb{R}^{k},|x|$ denotes the Euclidean norm of $x$. For any $k \times k$ matrix $A$, define the norm of $A$ by $|A|=\sup \{|A x|:|x| \leq 1\}$. Let $X$ denote the vector space of bounded sequences set $\phi:[0, \infty) \rightarrow \mathbb{R}^{k}$ and $\|\phi\|=\sup \{|\phi(s)|: s \geq 0\}$. For more on Volterra summation equations we refer to [2] and [10]. 


\section{Boundedness and Periodicity via Fixed Point Theory}

Lyapunov functionals combined with the resolvent and fixed point theory are used to obtain results concerning boundedness and existence of an asymptotically periodic solution. We begin with the following easy theorem regarding the existence of solutions of (1.3).

Theorem 2.1. Assume the existence of two positive constants $K$ and $\alpha \in(0,1)$ such that $a \in X$ we have

$$
|a(t)| \leq K \text { and } \sup _{t \geq 0} \sum_{s=0}^{t-1}|C(t, s)| \leq \alpha \text { for all } t \in \mathbb{Z}^{+}
$$

then there is a unique bounded solution of (1.3).

Proof. Define a mapping $D: X \rightarrow X$, by

$$
(D \phi)(t)=a(t)-\sum_{s=0}^{t-1} C(t, s) \phi(s) .
$$

It is clear that $(X,\|\cdot\|)$ is a Banach space. Now for $\phi \in X$, with $\|\phi\| \leq q$ for positive constant $q$ we have that

$$
\|(D \phi)\| \leq K+\alpha q .
$$

Thus $D: X \rightarrow X$. Left to show that $D$ defines a contraction mapping on $X$. Let $\phi, \varphi \in X$. Then

$$
\begin{aligned}
\|(D \phi)-(D \varphi)\| & \leq \sup _{t \geq 0} \sum_{s=0}^{t-1}|C(t, s)|\|\phi-\varphi\| \\
& \leq \alpha\|\phi-\varphi\| .
\end{aligned}
$$

Hence, $D$ is a contraction and by the contraction mapping principle it has a unique fixed point in $X$ that solves (1.3). This completes the proof.

In Theorem 3 of [3], the authors used lower and upper inequalities to trap the solutions of (1.3) and show that they are bounded. However, Theorem 2.1 provides conditions for the existence of a unique solution of (1.3) using simple mapping applied to the contraction mapping principle. We have the following theorem in which we use a Lyapunov functional to drive solutions to zero.

Theorem 2.2. Assume the existence of two positive constants $K_{1}$ and $\alpha \in(0,1)$ such that

$$
\sum_{t=0}^{\infty}|a(t)| \leq K_{1} \text { and } \sum_{u=1}^{\infty}|C(u+t, t)| \leq \alpha, \text { for all } t \in \mathbb{Z}^{+}
$$

then every solution $x(t)$ of (1.3) satisfies $x \in l_{1}[0, \infty)$ and $x(t) \rightarrow 0$, as $t \rightarrow \infty$. 
Proof. Using (1.3) we obtain

$$
|x(t)|-|a(t)| \leq \sum_{s=0}^{t-1}|C(t, s)||x(s)| .
$$

Define the Lyapunov functional $V$ by

$$
V(t)=\sum_{s=0}^{t-1} \sum_{u=t-s}^{\infty}|C(u+s, s)||x(s)| .
$$

Moreover,

$$
\triangle V(t)=|x(t)| \sum_{u=1}^{\infty}|C(u+s, s)|-\sum_{s=0}^{t-1}|C(t, s)||x(s)| .
$$

A substitution of (2.3) in the above expression yields

$$
\begin{aligned}
\triangle V(t) & \leq|x(t)| \sum_{u=1}^{\infty}|C(u+s, s)|-|x(t)|+|a(t)| \\
& =(\alpha-1)|x(t)|+|a(t)| .
\end{aligned}
$$

Summing the above inequality from 0 to $t-1$ gives

$$
0 \leq V(t) \leq V(0)+(\alpha-1) \sum_{s=0}^{t-1}|x(s)|+\sum_{s=0}^{t-1}|a(s)| .
$$

Since $V(0)=0$ and $\alpha \in(0,1)$ we arrive at

$$
\sum_{s=0}^{t-1}|x(s)| \leq \frac{1}{1-\alpha} \sum_{s=0}^{t-1}|a(s)| .
$$

Letting $t \rightarrow \infty$ we have

$$
\sum_{t=0}^{\infty}|x(t)| \leq \frac{1}{1-\alpha} \sum_{t=0}^{\infty}|a(t)| \leq \frac{1}{1-\alpha} K_{1},
$$

which implies that $x(t) \rightarrow 0$, as $t \rightarrow \infty$. This completes the proof.

We note that the use of Lyapunov functional has an advantage here due to the absence of a linear term in our original equation (1.3) which is necessary for the use of variation of parameters. The next result is about the existence of a unique periodic solution for the Volterra summation equation

$$
x(t)=a(t)-\sum_{s=-\infty}^{t-1} C(t, s) x(s), t \in \mathbb{Z}
$$

where $x$ and $a$ are $k$-vectors, $k \geq 1$, while $C$ is an $k \times k$ matrix. 
Theorem 2.3. Assume the existence of a constant $\alpha \in(0,1)$ such that

$$
\sup _{t \geq 0} \sum_{s=-\infty}^{t-1}|C(t, s)| \leq \alpha
$$

and there is a positive integer $T$ such that

$$
a(t+T)=a(t) \text { and } C(t+T, s+T)=C(t, s),
$$

then there is a unique periodic solution of (2.4).

Proof. Let $X$ be the space of periodic sequences of period $T$. Then, it is clear that $(X,\|\cdot\|)$ is a Banach space under the supremum norm. Now for $\phi \in X$, we define $D: X \rightarrow X$ by

$$
(D \phi)(t)=a(t)-\sum_{s=-\infty}^{t-1} C(t, s) \phi(s) .
$$

It is clear that $\mathrm{D}$ is periodic of period $T$. That is $(D \phi)(t+T)=(D \phi)(t)$. It remains to show that $D$ defines a contraction mapping on $X$. Let $\phi, \varphi \in X$. Then

$$
\begin{aligned}
\|(D \phi)-(D \varphi)\| & \leq \sup _{t \in \mathbb{Z}} \sum_{s=-\infty}^{t-1}|C(t, s)|\|\phi-\varphi\| \\
& \leq \alpha\|\phi-\varphi\| .
\end{aligned}
$$

Hence, $D$ is a contraction and by the contraction mapping principle it has a unique fixed point in $X$ that solves (2.4). This completes the proof.

In the next theorem we return to (1.3) and rewrite it so we can show it has an asymptotically periodic solution. Thus we rewrite (1.3) in the form

$$
x(t)=a(t)-\sum_{s=-\infty}^{t-1} C(t, s) x(s)+\sum_{s=-\infty}^{-1} C(t, s) x(s) .
$$

Note that the term $a(t)-\sum_{s=-\infty}^{t-1} C(t, s) x(s)$ produced a unique periodic solution as we have seen in Theorem 2.3 and this indicates that for any bounded $x$, the term $\sum_{s=-\infty}^{-1} C(t, s) x(s) \rightarrow 0, t \rightarrow \infty$. Hence it is intuitive to expect a solution $x$ of (2.5) to be written as $x=y+z$ where $y$ is periodic and $z \rightarrow 0$, as $t \rightarrow \infty$. We need to properly define our spaces. Let

$$
P_{T}=\left\{\phi: \mathbb{Z} \rightarrow \mathbb{R}^{k} \mid \phi(t+T)=\phi(t)\right\}
$$

and

$$
Q=\left\{q: \mathbb{Z}^{+} \rightarrow \mathbb{R}^{k} \mid q(t) \rightarrow 0, \text { as } t \rightarrow \infty\right\} .
$$

We have the following theorem. 
Theorem 2.4. Suppose for $\phi \in P_{T}$,

$$
\sum_{s=-\infty}^{-1} C(t, s) \phi(s) \rightarrow 0, \text { as } t \rightarrow \infty
$$

and for each $z \in Q$,

$$
\sum_{s=0}^{t-1} C(t, s) z(s) \rightarrow 0, \text { as } t \rightarrow \infty .
$$

Assume the existence of a constant $\alpha \in(0,1)$ such that

$$
\sup _{t \geq 0} \sum_{s=0}^{t-1}|C(t, s)| \leq \alpha
$$

and there is a positive constant integer $T$ such that $a(t+T)=a(t)$ and $C(t+T, s+$ $T)=C(t, s)$. Then (1.3) has a solution $x(t)=y(t)+z(t)$ where $y \in P_{T}$ and $z \in Q$.

Proof. Let $X$ be the space of sequences $\phi: \mathbb{Z}^{+} \rightarrow \mathbb{R}^{k}$ such that $\phi \in X$ implies there is a $y \in P_{T}$ and $z \in Q$ with $\phi=y+z$. We claim that $(X,\|\cdot\|)$ is a Banach space, where $\|\cdot\|$ is the supremum norm. To see this, we suppose $\left\{y_{n}+z_{n}\right\}$ is a Cauchy sequence in $(X,\|\cdot\|)$. Given an $\varepsilon>0$ there is an $\mathrm{N}$ such that for $n, m \geq N$ we have

$$
\left|y_{n}(t)+z_{n}(t)-y_{m}(t)-z_{m}(t)\right|<\frac{\varepsilon}{2} .
$$

Since $z \in Q$ we have that for each $\varepsilon>0$ and each $z \in Q$ there is an $L>0$ such that $t \geq L$, implies that $|z(t)| \leq \varepsilon / 4$. Fix $n, m \geq N$ and for the $\varepsilon / 4$ find $L>0$ such that $t \geq L$, implies that

$$
\left|y_{n}(t)-z_{m}(t)\right|-\left|z_{n}(t)-y_{m}(t)\right| \leq\left|y_{n}(t)+z_{n}(t)-y_{m}(t)-z_{m}(t)\right|<\frac{\varepsilon}{2} .
$$

Thus, for $t \geq L$ we arrive at

$$
\left|y_{n}(t)-y_{m}(t)\right| \leq \frac{\varepsilon}{2}+\left|z_{n}(t)\right|+\left|z_{m}(t)\right|<\varepsilon,
$$

for all $t$ since $y_{n}, y_{m} \in P_{T}$. Since this holds for every pair with $n \geq N$ and $m \geq N$, it follows that $\left\{y_{n}\right\}$ is a Cauchy sequence. The same argument can be repeated for the sequence $\left\{y_{n}\right\}$. This completes the proof of the claim.

Let $\phi=y+z$ where $y \in P_{T}$ and $z \in Q$. Define the mapping $H: X \rightarrow X$ by

$$
(H \phi)(t)=a(t)-\sum_{s=0}^{t-1} C(t, s) x(s) .
$$


Then $H$ is a contraction mapping by Theorem 2.3. Now we observe that

$$
\begin{aligned}
(H \phi)(t) & =a(t)-\sum_{s=0}^{t-1} C(t, s) \phi(s) \\
& =a(t)-\sum_{s=0}^{t-1} C(t, s)(y(s)+z(s)) \\
& =\left[a(t)-\sum_{s=-\infty}^{t-1} C(t, s) y(s)\right] \\
& +\left[\sum_{s=-\infty}^{-1} C(t, s) y(s)-\sum_{s=0}^{t-1} C(t, s) z(s)\right] \\
& :=B \phi+A \phi .
\end{aligned}
$$

This defines mappings $A$ and $B$ on $X$. Note that $B: X \rightarrow P_{T} \subset X$ and $A: X \rightarrow Q \subset$ $X$. Since $H$ defines a contraction mapping, it has a unique fixed point in $X$ that solves (1.3). The proof is complete.

In [5] the authors considered the Volterra difference equation

$$
x(n+1)=a(n)+b(n) x(n)+\sum_{i=0}^{n} C(n, i) x(i)
$$

and studied the existence of asymptotically periodic solution by inverting (2.6). The inversion was made possible due to the existence of the term $b(n) x(n)$ under the assumption that $b(n) \neq 0$ for all $n \in \mathbb{Z}^{+}$. Thus, no comparison can be made between this work and [5] and [9]. The same can be said about the paper [7]. The literature is vast regarding Volterra difference equations of the form (2.6), unlike Volterra summation equations of the form of (1.3).

\section{Resolvent and Lyapunov Functionals}

In this section we use the notion of resolvent to obtain a variation of parameters formula, coupled with Lyapunov functionals to arrive at various results concerning the solutions of

$$
x(t)=a(t)-\sum_{s=0}^{t-1} C(t, s) x(s),
$$

where all the terms are to be considered as defined in previous sections. In [12], Vecchio obtained the resolvent equation of (3.1) to be

$$
R(t, s)=C(t, s)-\sum_{u=s+1}^{t-1} R(t, u) C(u, s) .
$$


If $C$ is scalar valued, then so is $R$. If $C$ is $n \times n$ matrix, then so is $R$. Moreover, the solution of (3.1) in term of $R$ is given by

$$
x(t)=a(t)-\sum_{s=0}^{t-1} R(t, s) a(s) .
$$

Adivar et al., [1] were the first to extend the results of [12] to (1.3) on time scales.

For the remainder of this section we denote the vector space of bounded sequences $\phi: \mathbb{Z}^{+} \rightarrow \mathbb{R}^{k}$ by $\mathcal{B C}$. The authors in [1] extended Perron's Theorem, (see [11]) to time scale. Thus, by setting the time scale equal the set of integers $\mathbb{Z}$ we get the following special theorem.

Theorem 3.1. Let $C: \mathbb{Z}^{+} \times \mathbb{Z}^{+} \rightarrow \mathbb{R}$ be real valued sequence on $0 \leq t_{0} \leq s \leq t<\infty$. If $\sum_{s=0}^{t-1} R(t, s) f(s) \in \mathcal{B C}$ for each $f \in \mathcal{B C}$, then there exists a positive constant $K$ such that $\sum_{s=0}^{t-1}|R(t, s)|<K$, for all $t \in \mathbb{Z}^{+}$.

Theorem 3.2. Suppose $R(t, s)$ satisfies (3.2) and that $a \in \mathcal{B C}$. Then every solution $x(t)$ of (3.1) is bounded if and only if

$$
\sup _{t \in \mathbb{Z}^{+}} \sum_{s=0}^{t-1}|R(t, s)|<\infty
$$

holds.

Proof. Suppose (3.4) hold. Then, using (3.3), it is trivial to show that $x(t)$ is bounded. If $x(t)$ and $a(t)$ are bounded, then from (3.3), we have

$$
\sum_{s=0}^{t-1}|R(t, s)||a(s)| \leq \gamma
$$

for some positive constant $\gamma$ and the proof follows from Theorem 3.1.

The intuitive idea here is that for $C(t, s)$ well-behaved then the solution of (3.1) follows $a(t)$.

Theorem 3.3. Let $C$ be a $k \times k$ matrix. Assume the existence of a constant $\alpha \in(0,1)$ such that

$$
\sup _{t \in \mathbb{Z}^{+}} \sum_{s=0}^{t-1}|C(t, s)| \leq \alpha,
$$

(i) If $a \in \mathcal{B C}$ so is the solution $x$ of (3.1); hence, (3.5) holds. 
(ii) Suppose, in addition, that for each $T>0$ we have $\sum_{s=0}^{T}|C(t, s)| \rightarrow 0$ as $t \rightarrow \infty$. If $a(t) \rightarrow 0$ as $t \rightarrow \infty$, then so does $x(t)$ and $\sum_{s=0}^{t-1} R(t, s) a(s)$.

(iii) $\sum_{s=0}^{t-1}|R(t, s)| \leq \frac{\alpha}{1-\alpha}$.

Proof. The proof of $(i)$ is the same as the proof of Theorem 2.1. For the proof of $(i i)$ we define the set

$$
M=\left\{\phi: \mathbb{Z}^{+} \rightarrow \mathbb{R}^{k}|| \phi(t) \mid \rightarrow 0, \text { as } t \rightarrow \infty\right\} .
$$

For $\phi \in M$, define the mapping $Q$ by

$$
(Q \phi)(t)=a(t)-\sum_{s=0}^{t-1} C(t, s) \phi(s) .
$$

Then

$$
|(Q \phi)(t)| \leq|a(t)|+\sum_{s=0}^{t-1}|C(t, s) \phi(s)| .
$$

We already know that $a(t) \rightarrow 0$ as $t \rightarrow \infty$. Given an $\varepsilon>0$ and $\phi \in M$, find $T$ such that $|\phi(t)|<\varepsilon$ if $t \leq T$ and find $d$ with $|\phi(t)| \leq d$ for all $t \geq T$. For this fixed $T$, find $\eta>T$ such that $t \geq \eta$ implies that $\sum_{s=0}^{T-1}|C(t, s)| \leq \frac{\varepsilon}{d}$. Then $t \geq \eta$ implies that

$$
\begin{aligned}
\sum_{s=0}^{t-1}|C \phi(s)| & \leq \sum_{s=0}^{T-1}|C(t, s)||\phi(s)|+\sum_{s=T}^{t-1}|C(t, s)||\phi(s)| \\
& \leq(d \varepsilon) / d+\alpha \varepsilon<2 \varepsilon .
\end{aligned}
$$

Thus, $Q: M \rightarrow M$ and the fixed point satisfies $x(t) \rightarrow 0$, as $t \rightarrow \infty$, for every vector sequence $a \in M$. Using (3.3) we have

$$
\sum_{s=0}^{t-1} R(t, s) a(s)=a(t)-x(t) \rightarrow 0, \text { as } t \rightarrow \infty .
$$

This completes the proof of $(i i)$. Using (3.2) and (3.5) we have by changing of order of summations

$$
\sum_{s=0}^{t-1}|R(t, s)| \leq \sum_{s=0}^{t-1}|C(t, s)|+\sum_{s=0}^{t-1} \sum_{u=s+1}^{t-1}|R(t, u)||C(u, s)|
$$




$$
\begin{aligned}
& =\sum_{s=0}^{t-1}|C(t, s)|+\sum_{u=0}^{t-1}\left(|R(t, u)| \sum_{s=0}^{u-1}|C(u, s)|\right) \\
& \leq \alpha+\alpha \sum_{u=0}^{t-1}|R(t, u)| .
\end{aligned}
$$

Therefore,

$$
(1-\alpha) \sum_{s=0}^{t-1}|R(t, s)| \leq \alpha
$$

That is,

$$
\max _{t \in \mathbb{Z}^{+}} \sum_{s=0}^{t-1}|R(t, s)| \leq \frac{\alpha}{1-\alpha}
$$

This completes the proof.

Example 3.4. Suppose there is a sequence $r: \mathbb{Z}^{+} \rightarrow(0,1]$, with $r(t) \downarrow 0$ with

$$
\sup _{t \in \mathbb{Z}^{+}} \sum_{s=0}^{t-1}|C(t, s)|(r(s) / r(t)) \leq \alpha, \alpha \in(0,1)
$$

and

$$
|a(t)| \leq k r(t) \text { for all } t \in \mathbb{Z}^{+}
$$

for some positive constant $k$. Then the unique solution $x(t)$ of (3.1) is bounded and goes to zero as $t$ approaches infinity. Moreover, $\sum_{s=0}^{t-1} R(t, s) a(s) \rightarrow 0$, as $t \rightarrow \infty$.

Proof. Let

$$
\mathcal{M}=\left\{\phi:\left.[0, \infty) \rightarrow \mathbb{R}^{k}|| \phi\right|_{r}=\sup _{t \in \mathbb{Z}^{+}} \frac{|\phi(t)|}{r(t)}<\infty\right\} .
$$

Then $\left(\mathcal{M},|\cdot|_{r}\right)$ is a Banach space. For $\phi \in \mathcal{M}$, define the mapping $Q$ by

$$
(Q \phi)(t)=a(t)-\sum_{s=0}^{t-1} C(t, s) \phi(s) .
$$

Then,

$$
\begin{aligned}
|(Q \phi)(t)| / r(t) & \leq|a(t)| / r(t)+\sum_{s=0}^{t-1}|C(t, s)|(r(s) / r(t))|\phi(s)| / r(s) \\
& \leq k+|\phi|_{r} \sum_{s=0}^{t-1}|C(t, s)|(r(s) / r(t))
\end{aligned}
$$




$$
\leq k+\alpha|\phi|_{r},
$$

which shows that $Q \phi \in \mathcal{M}$. Let $\phi, \eta \in \mathcal{M}$, then we readily have that

$$
\mid(Q \phi)(t))-(Q \eta)(t)|/ r(t) \leq \alpha| \phi-\left.\eta\right|_{r}
$$

and so we have $Q$ is a contraction on $\mathcal{M}$ and therefore it has a unique fixed point $x(t)$ in $\mathcal{M}$ that solves (3.1). Moreover, $\sup _{t \in \mathbb{Z}^{+}} \frac{|x(t)|}{r(t)}<\infty$, implies that $|x(t)| \leq k^{*} r(t) \rightarrow 0$, as $t \rightarrow \infty$. Also by (3.7) we have $|a(t)| \rightarrow 0$, as $t \rightarrow \infty$ and hence using (3.3) we have

$$
\sum_{s=0}^{t-1}|R(t, s) a(s)| \leq|a(t)|+|x(t)| \rightarrow 0, \text { as } t \rightarrow \infty .
$$

This completes the proof.

The next theorem relate the kernel to a kernel of convolution type. Also, unlike Theorem 2.2 we only ask for boundedness on $a(t)$.

Theorem 3.5. Assume the existence of a constant $\alpha \in(0,1)$ such that

$$
\sum_{u=1}^{\infty}|C(u+t, t)| \leq \alpha
$$

and let $a(t)$ be a bounded sequence. Suppose there is a decreasing sequence $\Phi$ : $[0, \infty) \rightarrow(0, \infty)$ with $\Phi \in l_{1}[0, \infty)$, and

$$
\Phi(t-s) \geq \sum_{u=t-s}^{\infty}|C(u+s, s)| .
$$

If in addition, there exists a positive constant $K$ with

$$
\sum_{u=t-s}^{\infty}|C(u+s, s)| \geq K|C(t, s)|
$$

then the unique solution $x(t)$ of (3.1) is bounded and

$$
\sup _{t \in \mathbb{Z}^{+}} \sum_{s=0}^{t-1}|R(t, s)|<\infty .
$$

Proof. Define the Lyapunov functional $V$ by

$$
V(t)=\sum_{s=0}^{t-1} \sum_{u=t-s}^{\infty}|C(u+s, s)||x(s)| .
$$


Then along the solutions of (3.1) we have

$$
\triangle V(t)=|x(t)| \sum_{u=1}^{\infty}|C(u+s, s)|-\sum_{s=0}^{t-1}|C(t, s)||x(s)| .
$$

Using (3.1) we arrive at

$$
|x(t)|-|a(t)| \leq \sum_{s=0}^{t-1}|C(t, s)||x(s)| .
$$

Hence,

$$
\begin{aligned}
\triangle V(t) & \leq|x(t)| \sum_{u=1}^{\infty}|C(u+s, s)|-|x(t)|+|a(t)| \\
& =(\alpha-1)|x(t)|+|a(t)|:=-\delta|x(t)|+|a(t)|
\end{aligned}
$$

for $\delta>0$. Replace $t$ with $s$ in the above expression and then multiply both sides by $\Phi(t-s)$ for $0 \leq s \leq t<\infty$.

$$
\triangle_{s} V(s) \Phi(t-s) \leq-\delta|x(s)| \Phi(t-s)+|a(s)| \Phi(t-s) .
$$

Suppose there is a $t>0$ satisfying

$$
V(t)=\max _{0 \leq s \leq t-1} V(s+1) .
$$

Then summing from 0 to $t-1$ followed with summation by parts and by noting that $V(0)=0$ we arrive at

$$
\begin{aligned}
\sum_{s=0}^{t-1} \triangle_{s} V(s) \Phi(t-s) & =\left.V(s) \Phi(t-s)\right|_{s=0} ^{t}-\sum_{s=0}^{t-1} V(s+1) \triangle_{s} \Phi(t-s) \\
& \geq V(t) \Phi(0)-V(0) \Phi(t)-V(t) \sum_{s=0}^{t-1} \triangle_{s} \Phi(t-s) \\
& =V(t) \Phi(0)-V(t)[\Phi(0)-\Phi(t)] \\
& =V(t) \Phi(t) .
\end{aligned}
$$

Hence (3.12) combined with (3.11) and making use of (3.10) yield

$$
\begin{aligned}
V(t) \Phi(t) & \leq \sum_{s=0}^{t-1} \triangle_{s} V(s) \Phi(t-s) \\
& \leq-\delta \sum_{s=0}^{t-1} \Phi(t-s)|x(s)|+\sum_{s=0}^{t-1} \Phi(t-s)|a(s)|
\end{aligned}
$$




$$
\begin{aligned}
& \leq-\delta \sum_{s=0}^{t-1} \sum_{u=t-s}^{\infty}|C(u+s, s)||x(s)|+\sum_{s=0}^{t-1} \Phi(t-s)|a(s)| \\
& \leq-\delta V(t)+\|a\| q
\end{aligned}
$$

where $\|a\|$ is the supremum norm of $a$ and $q$ is a positive constant that we get from $|\Phi| \in l_{1}[0, \infty)$. The above inequality implies that

$$
V(t) \leq \frac{\|a\| q}{\Phi(t)+\delta}
$$

and $V(t)$ is bounded. Using (3.10) in $V(t)$ gives

$$
V(t) \geq K \sum_{s=0}^{t-1}|C(t, s)||x(s)| \geq K[|x(t)|-|a(t)|],
$$

from which we conclude $x(t)$ is bounded since both $V(t)$ and $a(t)$ are bounded. Now from (3.3) we have that $\sum_{s=0}^{t-1}|R(t, s) a(s)| \leq|x(t)|+|a(t)|$ and hence $\sum_{s=0}^{t-1}|R(t, s) a(s)|$ is bounded and by Theorem 3.1, we have that $\sup _{t \in \mathbb{Z}^{+}} \sum_{s=0}^{t-1}|R(t, s)|<\infty$. This completes the proof.

For Theorem 3.6 we assume (3.1) is scalar.

Theorem 3.6. Assume the existence of constants $\alpha, \beta \in(0,1)$ such that

$$
\sum_{u=1}^{\infty}|C(u+t, t)| \leq \alpha \text { for all } t \in \mathbb{Z}^{+}
$$

and

$$
\sup _{t \in \mathbb{Z}^{+}} \sum_{s=0}^{t-1}|C(t, s)| \leq \beta
$$

If $a \in l_{2}[0, \infty)$ so is the solution $x$ of (3.1).

Proof. Define the Lyapunov functional $V$ by

$$
V(t)=\sum_{s=0}^{t-1} \sum_{u=t-s}^{\infty}|C(u+s, s)| x^{2}(s) .
$$

Then along the solutions of (3.1) we have that

$$
\triangle V(t)=x^{2}(t) \sum_{u=1}^{\infty}|C(u+s, s)|-\sum_{s=0}^{t-1}|C(t, s)| x^{2}(s) .
$$


Squaring both sides of (3.1) gives

$$
\begin{aligned}
x^{2}(t) & =a^{2}(t)-2 a(t) \sum_{s=0}^{t-1} C(t, s) x(s)+\left(\sum_{s=0}^{t-1} C(t, s) x(s)\right)^{2} \\
& \leq 2\left(a^{2}(t)+\left(\sum_{s=0}^{t-1} C(t, s) x(s)\right)^{2}\right) \\
& \leq 2 a^{2}(t)+2 \sum_{s=0}^{t-1}|C(t, s)| \sum_{s=0}^{t-1}|C(t, s)| x^{2}(s) \\
& \leq 2 a^{2}(t)+2 \beta \sum_{s=0}^{t-1}|C(t, s)| x^{2}(s) .
\end{aligned}
$$

This implies that

$$
-\sum_{s=0}^{t-1}|C(t, s)| x^{2}(s) \leq a^{2}(t) / \beta-x^{2}(t) /(2 \beta) .
$$

Substituting into $\triangle V$ gives

$$
\begin{aligned}
\triangle V(t) & =x^{2}(t) \sum_{u=1}^{\infty}|C(u+s, s)|-\sum_{s=0}^{t-1}|C(t, s)| x^{2}(s) \\
& \leq a^{2}(t) / \beta-(1 /(2 \beta)-\alpha) x^{2}(t) .
\end{aligned}
$$

Summing the above inequality for 0 to $n-1$ yields

$$
0 \leq V(t)-V(0) \leq 1 / \beta \sum_{s=0}^{n-1} a^{2}(s)-(1 /(2 \beta)-\alpha) \sum_{s=0}^{n-1} x^{2}(s),
$$

and hence the results. This completes the proof.

\section{References}

[1] M. Adivar, and Y. Raffoul, Existence of resolvent for Volterra integral equations on time scales, Bull. Aust. Math. Soc. 82 (2010), 139-155.

[2] M. Adivar, and Y. Raffoul, Qualitative analysis of nonlinear Volterra integral equations on time scales using resolvent and Lyapunov functionals, Applied Mathematics and Computation 273 (2016) 258-266.

[3] J. Appleby, I. Györi, , and D. Reynols. On exact convergence rates for solutions of linear systems of Volterra difference equations, Journal of Difference Equations and Applications, 12(12):1257-1275, 2006. 
[4] M. Bohner, and A. Peterson, Dynamic Equations on Time Scales, An Introduction with Applications, Birkhäuser, Boston, 2001.

[5] J. Diblik, M. Ruzickova, E. Schmeidel, and M. Zbaszyniak. Weighted asymptotically periodic soluions of linear Volterra difference equations, Abstract and Applied Analysis, Volume 2011.

[6] S. Elaydi, An Introduction to Difference Equations, Springer Verlag, New York, 3rd Edition, 2005.

[7] I. Györi, and D. Reynols, On asymptotically periodic solutions of linear discrete Volterra equations, Fasciculi Mathematici, 44(1):53-67, 2010.

[8] W. Kelley, and A. Peterson, Difference Equations An Introduction With Applications, Second Edition, Academic Press, New York, 2001.

[9] V. Kolmanovskii, and L. Shaikhet, Some conditions for boundedness of solutions of difference Volterra equations, Applied Mathematics Letters, 16(6):857-862, 2003.

[10] R. Medina, Solvability of discrete Volterra equations in weighted spaces, Dynamic Systems and Appl. 5(1996), 407-422.

[11] O. Perron, Die Stabilitätsfrage bei Differentialgleichungen, (1930) Math. Z. 32, PP:703-728. Dynamic Systems and Appl. 5(1996), 407-422.

[12] A. Vecchio, On the resolvent kernel of Volterra discrete equation, Functional Differential Equations, 6(1-2): p:191-201, 1999.

[13] S. Zhang, Stability of neutral delay difference systems, Computers Math. Applic. (2001) Vol. 42, pp. 291-299. 